\title{
Los colombianos se comprometen a cuidar el corazón
}

\author{
Colombians are committed to taking care of the heart
}

\author{
Alexis Llamas-Jiménez¹, Jaime Calderón-Herrera², Leany Blandón-Rentería y Juan C. Santacruz* \\ ${ }^{1}$ Servicio de Cardiología, Clínica de las Américas, Medellín; ${ }^{2}$ Fundación Colombiana del Corazón, Bogotá. Colombia
}

\section{Resumen}

Como una respuesta a la realidad epidemiológica del crecimiento de la mortalidad a causa de las enfermedades cardiocerebrovasculares en el país, la Fundación Colombiana del Corazón (FCC) decidió, como estrategia, incentivar en la comunidad el cuidado como estilo de vida, y para ello propuso movilizar a los colombianos para que realizaran promesas para el cuidado de su corazón. El objetivo de este artículo es mostrar cómo se realizó la implementación de esta estrategia y revisar los resultados de las promesas hechas por los colombianos para cuidar su corazón en septiembre de 2018, denominado el "Mes del corazón". Más de 30.000 compromisos se lograron en todo el país con la estrategia de comunicación denominada "Mes del corazón, haz tu promesa" mediante un instrumento que indagó por los compromisos de las personas para el cuidado de su corazón. La FCC identificó cincuenta posibles promesas obtenidas de los comportamientos planteados por la propuesta institucional de generación propia, conocida como "Corazones responsables". Se procesaron 2.412 promesas (encuestas) obtenidas en el marco de los eventos académicos y de empresas programados por la FCC y la Sociedad Colombiana de Cardiología y Cirugía Cardiovascular, y de cuya tabulación pudieron identificarse los compromisos comúnmente propuestos por la comunidad. Entre los más destacados, los colombianos se comprometieron a: 1. caminar y hacer más ejercicio, 2. tener pensamientos positivos, 3. dormir más (este más frecuente en mujeres), 4. aumentar el consumo de agua durante el día, 5. incrementar el consumo de frutas y verduras y 6. desayunar todos los días en forma saludable.

Palabras clave: Estilos de vida. Alimentación saludable. Promoción de la salud. Cuidado de la salud.

\section{Abstract}

As a response to the epidemiological reality of the growth of mortality from cardio-cerebrovascular diseases in our country, the Colombian Heart Foundation (FCC) decided as a strategy to encourage "care as a lifestyle" in the community and to this end proposed to mobilize Colombians to make promises for the care of your heart. The objective of this article is to present how the implementation of this strategy was carried out and to review the results of the promises made by Colombians to care for their hearts in September 2018, called Heart Month. More than 30 thousand commitments were achieved throughout the country with the communication strategy called "Month of the heart, make your promise" through an instrument that investigated the commitments of people to care for their hearts. The FCC identified 50 possible promises, obtained from the behaviors raised by the institutional proposal of its own generation, known as "Responsible hearts". 2412 promises (surveys) obtained within the framework of the academic and business events programmed by the FCC and the Colombian Society of

\section{Correspondencia:}

*Juan C. Santacruz

E-mail: jcsantacruz @ corazonesresponsables.org.
Fecha de recepción: 22-01-2019

Fecha de aceptación: 17-06-2020

DOI: 10.24875/RCCAR.M21000007
Disponible en internet: 19-03-2021 Rev Colomb Cardiol. 2021;28(1):38-44 www.rccardiologia.com 0120-5633 / C 2020 Sociedad Colombiana de Cardiología y Cirugía Cardiovascular. Publicado por Permanyer. Este es un artículo open access bajo la licencia CC BY-NC-ND (http://creativecommons.org/licenses/by-nc-nd/4.0/). 
Cardiology and Cardiovascular Surgery were processed and whose tabulation we were able to identify the commitments most frequently proposed by the community. Among the most prominent, Colombians committed to: 1. walking and exercising more, 2. having positive thoughts, 3. sleeping more (this is more frequent in women), 4. increasing water consumption during the day, 5. to increase the consumption of fruits and vegetables and 6. to eat breakfast every day in a healthy way.

Key words: Lifestyle. Healthy eating. Health promotion. Health care.

\section{Introducción}

Según cifras de la Organización Mundial de la Salud (OMS) de 2015, las enfermedades del corazón cobran al año 17,5 millones de vidas, siendo calificadas como el primer asesino del mundo'.

La Federación Mundial del Corazón, con el apoyo de la Organización Mundial de la Salud, inició la celebración del "Día mundial del corazón", como una estrategia global para generar mejor conocimiento sobre el riesgo de las enfermedades del corazón y acerca de la forma de controlarlas y prevenirlas. El 29 de septiembre del 2000 se celebró el primer "Día mundial del corazón" en más de cien países ${ }^{2-4}$.

En Colombia, la Sociedad Colombiana de Cardiología y Cirugía Cardiovascular y la Fundación Colombiana del Corazón, miembros activos de la Federación Mundial del Corazón, estructuraron desde 2010, el programa nacional de estilos de vida, denominado "Corazones responsables", que es una propuesta pedagógica de desarrollo original para la apropiación del cuidado como estilo de vida ${ }^{5}$.

Vivir la cultura del cuidado es aprender a comer, ser una persona activa, preferir espacios libres de humo, impulsar pensamientos positivos, estar aquí y ahora y disfrutar de la vitamina $\mathrm{N}$, de manera que el estilo de vida de una persona pueda ser ejemplo de donde vive, trabaja, estudia o se divierte.

La Fundación Colombiana del Corazón, como líder orientador de la estrategia, consideró en 2013 que era prudente ampliar el "Día mundial del corazón" a todo el mes, para tener mejor y más amplio impacto, generar mayor sensibilización e impulsar múltiples actividades destinadas a la toma de conciencia sobre la importancia del cuidado de la vida ${ }^{6}$.

Desde entonces, la Fundación homologó el planteamiento global de la Federación Mundial del Corazón al contexto nacional, generando sus propias piezas de comunicación, adaptando el lenguaje y proponiendo actividades propias con eventos académicos, lúdicos y de reflexión, que se orientan, a través de un kit, a la celebración del "Mes del corazón", el cual se comparte con colegios, universidades y empresas que hacen parte de los aliados que, en el ámbito nacional, ha impulsado la entidad?.

El objetivo fue establecer, desde el planteamiento conceptual de la Cultura del Cuidado de Corazones Responsables (planteamiento original de la Fundación Colombiana del Corazón), cuáles deben ser los elementos clave de comportamiento para priorizar las estrategias de promoción que realiza la entidad y que sirvan como insumo para comparativos e intervenciones a partir de las preferencias identificadas por la población a partir de una muestra aleatoria.

Este artículo se escribió con el fin de mostrar cómo se realizó la implementación de la estrategia de fomento del cuidado como estilo de vida y revisar los resultados de las promesas hechas por los colombianos para cuidar su corazón en septiembre de 2018, durante el "Mes del corazón".

\section{Materiales y métodos}

Para 2018, la Federación Mundial propuso impulsar una promesa en las personas, para que hagan un compromiso con su corazón con la campaña "Mi corazón, tu corazón". La Fundación Colombiana del Corazón adaptó la campaña a Colombia y en el mes y día del corazón se les pidió a las personas "hacer una promesa, de mi corazón, para el corazón, para todos nuestros corazones. Una promesa que el corazón agradezca porque es una muestra de amor"8-11.

La propuesta de la Federación Mundial fue abierta para que las personas hicieran la promesa que desearan. En la Fundación Colombiana del Corazón se estructuró un sondeo de selección múltiple entre las cincuenta alternativas planteadas. Se trata de cincuenta posibilidades de promesas que son el producto de comportamientos y conductas que protegen la vida y que están enmarcados en el "Método 10 del cuidado como estilo de vida", estrategia original del programa nacional de "Corazones responsables"10.

Mediante la encuesta diseñada para tal efecto, se realizó y se planteó la siguiente pregunta: ¿Cuáles son los compromisos que identifican las personas como 
prioritarios para lograr cuidar su corazón y preservar la salud?

\section{Caracterización de la encuesta}

El listado de las cincuenta promesas fue producto de una selección de comportamientos de vida que responden a los diez escenarios de cultura que aborda el Método 10, que contiene la cultura del movimiento, la de aprender a comer, la de espacios libres de humo, la de actuar con corazón de mujer, la del pensamiento positivo, la de la conciencia plena, la de la vitamina $\mathrm{N}$, la del cuidado en vacaciones, la de los números del corazón y la de organizaciones saludables. Para cada lineamiento de cultura se generaron propuestas de promesas. La lista de promesas se construyó con afirmaciones que proporcionan respuestas cerradas de selección múltiple. La libertad de las personas se circunscribió a elegir una o varias de las opciones de promesas que se les ofrecieron.

La encuesta se estructuró mediante la opción de selección múltiple, para hacerla versátil e intuitiva, y para que pueda generar datos claros y fáciles de analizar.

Es una lista fija de opciones de respuesta que brinda respuestas estructuradas y facilita la selección. Es importante señalar, no obstante, que los datos que se obtienen están limitados a las cincuenta opciones que se brindan.

La iniciativa "Haz tu promesa" hizo parte de un contexto de planeación y proyección de publicidad y mercadeo en Internet, redes sociales y correos electrónicos, mediante piezas de comunicación expresadas en afiches, hojas de promesas, tríptico informativo y post para redes sociales ${ }^{11}$.

La recolección de datos se hizo directamente, a través de los aliados, o por funcionarios de la Fundación, teniendo como instrumento de recogida de datos (encuesta) el formato físico, previamente seleccionado por las personas con la característica del anonimato de los sujetos.

La medición y construcción de los índices y escalas en la tabulación estuvieron estructuradas con el listado de promesas y la repetición de cada una según los datos de las planillas físicas.

La metodología de respuesta múltiple se planteó para que las personas pudieran elegir entre las cincuenta opciones que se les ofrecieron. Por esta razón, el desglose para la tabulación tiene cincuenta variables dicotómicas que son las mismas opciones planteadas.
Se tabuló cada variable dicotómica por 0 cuando no fue elegida y por 1 cuando fue elegida.

La metodología de medición fue la de sumar cada selección y traducirla a porcentajes a partir de considerar el $100 \%$ del total de las respuestas para distribuirlas porcentualmente en cada una de las cincuenta promesas propuestas. Los porcentajes representan la cantidad de personas que escogieron cada promesa como una proporción del número de personas que hicieron la selección.

En la tabla 1 se presenta la selección y la distribución porcentual de las propuestas seleccionadas en orden descendente con la distribución de frecuencia de selección porcentual de cada una de las opciones. La suma de los porcentajes correspondientes es mayor que 100, ya que cada persona que respondió podía elegir más de una opción.

Es importante señalar que el objetivo de la tabulación se orientó a obtener las frecuencias con las que cada opción fue elegida, sin tener en cuenta la elección combinada de 2, 3 o más por cada persona al considerarlo no relevante para responder la pregunta base del sondeo.

\section{Selección y evaluación de los participantes}

Diversas estrategias en el mundo se han planteado para favorecer la promoción de la salud. El esfuerzo más reciente se sintetizó con los objetivos del desarrollo sostenible, en los cuales se busca lograr un impacto en los determinantes que condicionan la salud de los seres humanos ${ }^{12}$.

En consecuencia, se han evidenciado estudios de salud laboral ${ }^{13,14}$, en los que se demuestra la importancia del reconocer el bienestar laboral como determinante fundamental del cuidado de la vida y de la salud. La vida del trabajo es un escenario pedagógico de gran valor para la toma de conciencia y el aprendizaje de comportamientos de estilo de vida orientados a preservar la salud. Por tal motivo, uno de los focos de la solicitud de promesas fueron las empresas que hacen parte del Programa de Certificación de Organizaciones Saludables de la Fundación Colombiana del Corazón (sistema de gestión de calidad con una guía de certificación cuyo alcance son la enfermedad cardiovascular y otras no transmisibles).

La Fundación Colombiana del Corazón desarrolló la propuesta de "Haz tu promesa" y la propuso a las empresas, universidades y colegios que hacen parte de sus aliados, con una breve guía metodológica, 
Tabla 1. Consolidación y tabulación de los compromisos con resultados porcentuales de las cincuenta promesas realizadas

Promesa

1 Voy a caminar más pasos al día.

2 Tendré en cuenta que nada ni nadie cambia, solo puedo hacerlo yo. Decidir tener pensamientos positivos sobre la gente y las situaciones.

3 Soy mujer y voy a ser ejemplo con mi estilo de vida para quienes me rodean.

$4 \quad$ Voy a asegurarme de dormir de 7 a 8 horas todos los días.

$5 \quad$ Voy a fomentar en mí los pensamientos positivos.

$6 \quad$ Voy a aumentar el agua al menos a 5 vasos cada día.

$7 \quad$ Voy a comer más frutas y verduras todos los días.

8 Voy a desayunar todos los días.

9 Voy a programar vacaciones para descansar y no para cansarme más.

10 Voy a mantenerme en el presente para tener siempre conciencia plena de lo que hago, siento, expreso y vivo.

11 Voy a buscar una actividad deportiva que me guste para disfrutar con amigos.

12 Voy a impulsar que mi empresa se convierta en una Organización Saludable.

13 Voy a intentar descubrir que el café sabe a café y no a azúcar.

14 No volveré a tomar jugos con azúcar.

17 Voy a ponerle sal a la comida solo después de probarla.

18 Voy a programar visitar al médico al menos una vez al año.

19 Voy a evitar las bebidas gaseosas.

20 Voy a rescatar el comedor de mi casa para que se convierta en un lugar de encuentro familiar.

21 Si voy a comer pediré asado, cocido o a la parrilla para evitar los fritos.

22 Voy a pararme de mi escritorio cada hora y voy a hacer 3 minutos de estiramientos.

23 Intentaré comer porciones pequeñas más veces al día, al menos cada 3 horas.

24 Voy a hacer silencio todos los días y me voy a entrenar hasta sumar 5 minutos de silencio conmigo mismo.

25 Cuando vaya a un restaurante voy a pedir comida baja en sal.

$26 \quad$ No le volveré a poner sal a las verduras.

27 Voy a bajar la sal en la preparación de mis comidas y la voy a cambiar por especias y condimentos.

29 Voy a disminuir los fritos a solo uno en la semana.

30 Voy a reducir a un máximo de 2 horas al día mi tiempo frente al televisor.

31 Voy a dinamizar mis sentidos para disfrutar de la naturaleza observando, oyendo, tocando, sintiendo.

32 Voy a programar un parque lleno de verde todos los fines de semana para nutrir mi mente y mi cuerpo.

34 Voy a comer todos los días grasas buenas, como pescado, aguacate o nueces.

35 Voy a evitar las comidas rápidas y mejor llevaré almuerzo preparado en casa para la oficina. 
Tabla 1. Consolidación y tabulación de los compromisos con resultados porcentuales de las cincuenta promesas realizadas (Continuación)

\begin{tabular}{|l|l|l|}
\hline 36 & Voy a mantener una mente curiosa para tener la disposición de descubrir algo nuevo todos los días. & $\%$ \\
\hline 37 & Voy a mercar más productos naturales que procesados. & 0.4 \\
\hline 38 & Voy a tomarme la presión arterial al menos una vez cada 6 meses. \\
\hline 39 & Voy a dejar el auto en el lugar más lejano del parqueadero para obligarme a caminar. & 0.4 \\
\hline 40 & Voy a ofrecerme todos los días a caminar 30 minutos con la mascota del hogar. \\
\hline 41 & Voy a programar hacerme perfil lipídico (colesterol y triglicéridos) cada año. & 0.3 \\
\hline 42 & Cuando coma carne le voy a quitar la grasa antes de comerla. \\
\hline 43 & Voy a aprender a elegir cuando hago mercado, cuando ordeno en un restaurante, cuando selecciono la comida en una & 0.2 \\
\hline 44 & Voy a aumentar la fibra diaria con más avena, frutas, verduras, fríjoles, lentejas, cebada y cereales. \\
\hline 45 & Voy a bajarme del transporte público tres cuadras antes para caminar de llegada a la oficina, al colegio 0 a la \\
\hline 46 & Voy a bajarme un piso antes del ascensor para subir escaleras. \\
\hline 47 & Voy a comprar alimentos sin grasas, especialmente la leche y sus derivados. \\
\hline 48 & Voy a contestar el teléfono siempre de pie. & 0.2 \\
\hline 49 & Voy a dejar de afectar con mi humo a los demás. \\
\hline 50 & Voy a hacer al menos una reunión de trabajo de pie. \\
\hline
\end{tabular}

respaldada por las piezas de comunicación. Adicionalmente, se aplicó la metodología del sondeo con las promesas en los eventos que hicieron parte del calendario académico de la Fundación.

Se trata, por tanto, de una muestra aleatoria, cuyo resultado son el número de planillas recibidas, válidas, procesadas y susceptibles de tabulación, las cuales fueron 2.412 en total.

Los participantes fueron los siguientes:

- Seminario "Cultura del cuidado", con la Universidad de Santander. Ciudad de Valledupar, capital del departamento de Cesar.

- Seminario "Actúa con corazón de mujer" con la Universidad de Boyacá. Ciudad de Tunja, capital del departamento de Boyacá.

- "Congreso nacional de estilos de vida", impulsado por la Secretaría de Salud de Medellín. Ciudad de Medellín, capital del departamento de Antioquia.

- Santuario de Fauna y Flora Otún Quimbaya. Ciudad de Pereira, capital del departamento de Risaralda.

- Secretaría de Salud de Medellín. Ciudad de Medellín, capital del departamento de Antioquia.

- "Simposio de Cardiología". Bucaramanga, capital del departamento de Santander.
- "Foro Internacional de Gestión de Ecoturismo". Jardín Botánico de Bogotá, capital de Colombia.

- Secretaría de Salud Municipal de Duitama. Ciudad de Duitama en el Departamento de Boyacá.

- Facultad de Medicina de la Universidad Antonio Nariño. Bogotá, capital de Colombia.

- Superintendencia de Industria y Comercio. Bogotá, capital de Colombia.

- Instituto Cardiovascular del Cesar. Ciudad de Valledupar, capital del departamento de Cesar.

- Cerrejón. Ciudad de Albania, departamento de la Guajira.

- Clínica de las Américas. Ciudad de Medellín, capital del departamento de Antioquia.

- Secretaría de Salud Departamental de Risaralda. Ciudad de Pereira, capital del departamento de Risaralda.

- Fundación Salud. Bogotá, capital de Colombia.

- Federación Nacional de Cafeteros, con los Comités de Cafeteros de 16 ciudades del país.

- Universidad del Sinú. Ciudad de Cartagena de Indias, capital del departamento de Bolívar.

- Universidad Corpas. Bogotá, capital de Colombia. 
- Clínica San José. Ciudad de Cúcuta, capital del departamento de Norte de Santander.

- Universidad de Nariño. Ciudad de Pasto, capital del departamento de Nariño.

\section{Resultados}

El trabajo de consolidación de las promesas permitió obtener un registro de 2.412 formularios válidos, los cuales fueron tabulados para establecer el orden de prioridades elegidas por los colombianos en las 28 ciudades del país que aceptaron la invitación y que enviaron el reporte de las promesas.

En el "Mes del corazón" los colombianos se comprometieron a caminar más, a tener pensamientos positivos, a ser ejemplo las mujeres, a dormir más, a aumentar la cantidad consumo de agua al día, a incrementar el consumo de frutas y verduras y a desayunar todos los días.

Los resultados evidencian las principales preocupaciones de las personas respecto de sus estilos de vida y los comportamientos que consideran más viables para conseguir impactar de manera positiva en la salud de sus corazones. Las condiciones de vida asociadas a la conservación de la salud mental son la prioridad fundamental identificada por quienes hicieron la selección de las promesas.

\section{Resultados}

- La mayor preocupación no está en lo físico, sino en lo mental. Un importante porcentaje priorizó los pensamientos positivos como compromiso, y la promesa que más se destacó fue la de caminar más a diario. Los comportamientos sedentarios preocupan de manera significativa a los colombianos.

- En cuanto a la comida, lo que más preocupa está asociado con la poca costumbre de hidratarse y por eso muchos se comprometieron a aumentar el consumo de agua.

- También las personas consideran que no están consumiendo suficientes frutas y verduras y prometieron hacerlo.

- La costumbre de salir corriendo al trabajo o al estudio sin desayuno es otra gran preocupación. Por eso también se priorizó la promesa de desayunar todos los días.

- Para las mujeres la propuesta de ser ejemplo con su estilo de vida fue definitiva y en un porcentaje significativo se comprometieron convertirse en ejemplo dentro de su entorno.
- Es curioso cómo los fines de semana y las vacaciones están en la clasificación del descanso cuando se les pregunta a las personas, pero en realidad el descanso es mínimo, pues la costumbre es comer más y dormir más en los días libres. Por eso es interesante resaltar que se haya escogido entre las prioridades comprometerse a descansar en las vacaciones y no cansarse más.

- Tener el descanso diario adecuado es otra notable preocupación. Por eso se priorizó la promesa de alcanzar las 7 a 8 horas diarias de sueño.

- Intentar estar aquí y ahora con la conciencia plena, también fue una de las promesas más relevantes, demostrando que existe una alta preocupación por las condiciones que generan estrés.

- Establecer relaciones a través de la práctica de actividad física también fue seleccionada como una de las prioridades. Muchos se comprometieron a buscar amigos para hacer alguna actividad deportiva.

- Muchos trabajadores están significativamente preocupados con que su entorno laboral permita decisiones saludables, por eso también se comprometieron a lograr que sus empresas se conviertan en organizaciones saludables y a que haya certificación de productos con características cardioprotectoras.

\section{Discusión}

La metodología aplicada es novedosa, no tomó sondeos aplicados anteriormente o con evidencia y no admitió contraste con otras similares, dada la originalidad de la propuesta como un caso de indagación experimental.

Los resultados obtenidos son la tabulación simple de las propuestas aceptadas y seleccionadas por las personas que recibieron el listado de las cincuenta promesas y quienes de manera libre seleccionaron aquellas con las que mejor se identificaron para lograr el cuidado de su corazón.

Este sondeo tiene limitaciones en la plena identificación y clasificación de los participantes y en la ausencia de una metodología de selección de las promesas con criterios de evidencia, ya que no se identificaron estudios similares.

La consolidación de los resultados genera un interés específico de conocimiento de las prácticas de comportamiento que las personas identifican como más viables para cuidar su corazón y se convierten en un insumo para el diseño de estrategias específicas de promoción, impulso y actividades de estilos de vida orientadas a la preservación de la salud del corazón. 
Los resultados son interesantes como referencia para estrategias puntuales de promoción de estilos de vida orientados al bienestar en entornos empresariales y educativos. De la misma forma, pueden ser utilizados en análisis comparativos o colaborativos tanto en el ámbito nacional como en el internacional.

\section{Conclusiones}

La solicitud de hacer una promesa de corazón tuvo una amplia respuesta de aceptación en los entornos empresariales y educativos que hacen parte del catálogo de alianzas impulsadas y establecidas por la Fundación Colombiana del Corazón.

Los resultados de la tabulación del sondeo son indicadores importantes de las tendencias que se identifican como claves para el cuidado del corazón, y señalan la importancia de aumentar los minutos de caminata diaria y la preservación de la salud mental como las más aceptadas para lograrlo.

\section{Conflicto de intereses}

Los autores y entidades que realizan este artículo declaran que no tienen conflicto de intereses que pudiera sesgar este trabajo.

\section{Responsabilidades éticas}

Protección de personas y animales. Los autores declaran que para esta investigación no se han realizado experimentos en seres humanos ni en animales.

Confidencialidad de los datos. Los autores declaran que en este artículo no aparecen datos de pacientes.

Derecho a la privacidad y consentimiento informado. Los autores declaran que en este artículo no aparecen datos de pacientes.

\section{Bibliografía}

1. World Health Organization. Enfermedad pulmonar obstructiva crónica (EPOC). [Internet]. 2017 [cited 20 November 2018]. Disponible en: https://www.who.int/es/news-room/fact-sheets/detail/cardiovascular-diseases-(cvds).

2. World Health Organization. World Heart Day. [Internet]. [cited 20 November 2018]. Disponible en: https://www.who.int/cardiovascular_diseases/ world-heart-day/en/.

3. World Heart Federation. WORLD HEART DAY. [Internet]. [cited 21 November 2018]. Disponible en: https://www.world-heart-federation.org/ world-heart-day/about-whd/.

4. Linn L. Día Mundial del Corazón: Adoptar hábitos saludables desde la infancia puede prevenir enfermedades del corazón. Panamerican Health Organization / World Health Organization. [Internet]. 2013 [cited 20 November 2018]. Disponible en: https://www.paho.org/hq/index.php?option=com_content $\&$ view=article $\& i d=9046: 2013$-dia-mundial-corazon-adoptar-habitos-saludables-infancia-prevenir-enfermedades-corazon\&ltemid $=1926 \&$ lang $=f r$.

5. Fundación Colombiana del Corazón. Corazonesresponsables.org. [Internet]. [cited 22 November 2018]. Disponible en: http://corazonesresponsables.org/

6. Santacruz J Corazones responsables para generar una cultura del cuidado. Portafolio.co [Internet]. 2014 [cited 21 November 2018]. Disponible en: https://www.portafolio.co/tendencias/corazones-responsables-generar-cultura-cuidado-55896.

7. Fundación Colombiana del Corazón. Corazonesresponsables.org. [Internet]. [cited 21 November 2018]. Disponible en: http://corazonesresponsables.org/blog/.

8. Santacruz J. Usar las escaleras y 49 promesas más para cuidar tu corazón. El universal [Internet]. www.eluniversal.com.co. 2018 [cited 21 November 2018]. Disponible en: https://www.eluniversal.com.co/salud/ usar-las-escaleras-y-49-promesas-mas-para-cuidar-tu-corazon-287848EUEU405076.

9. Santacruz J. Las promesas que le debe hacer a su corazón. EL HERALDO [Internet]. 2018 [cited 22 November 2018]. Disponible en: https:// www.elheraldo.co/salud/las-promesas-que-le-debe-hacer-su-corazon-548060.

10. Santacruz J. Al año mueren 17.5 millones de personas por enfermedades cardiovasculares, no sea una más. Bogota.extra.com.co. [Internet]. 2018 [cited 20 November 2018]. Disponible en: http://bogota.extra.com.co/ noticias/ciencia/salud/al-ano-mueren-175-millones-de-personas-por-enfermedades-car-464793.

11. Santacruz J. Una promesa en el mes del corazón: aprender a cuidarse para tener una vida mejor | ELESPECTADOR.COM [Internet]. 2018 [cited 21 November 2018]. Disponible en: https://www.elespectador.com/cromos/estilo-de-vida/una-promesa-en-el-mes-del-corazon-aprender-cuidarse-para-tener-una-vida-mejor-articulo-811463.

12. Grupo de Desarrollo Sostenible de las Naciones Unidas para América Latina y el Caribe. Desarrollo sostenible en América Latina y el Caribe: desafíos y ejes de política pública. Panamá, UNSDG. [Internet]. 2018 [Consultado 20 Nov 2018]. Disponible en: https://onu.org.gt/objetivos-de-desarrollo/.

13. Benavides FG, Delclós J, Serra C. El estado de bienestar y la salud pública. Elsevier [Internet]. 2018. [Consultado 20 Nov 2018]. 32(4): 377 380. Disponible en: https://www.sciencedirect.com/science/article/pii/ S0213911117301863.

14. González RE, Dardetb AC, López LA. Hacia una salud pública orientada desde los determinantes sociales de la salud: informe de un taller. Elsevier [Internet]. 2018. [Consultado 20 Nov 2018]. 32(5): 488-491. Disponible en: https://www.sciencedirect.com/science/article/pii/S0213911117302212. 